PROCEEDINGS OF THE

AMERICAN MATHEMATICAL SOCIETY

Volume 130, Number 3, Pages 915-923

S 0002-9939(01)06158-5

Article electronically published on October 1, 2001

\title{
THE POINT SPECTRUM OF THE DIRAC OPERATOR ON NONCOMPACT SYMMETRIC SPACES
}

\author{
S. GOETTE AND U. SEMMELMANN
}

(Communicated by Rebecca Herb)

\begin{abstract}
In this note, we consider the Dirac operator $D$ on a Riemannian symmetric space $M$ of noncompact type. Using representation theory, we show that $D$ has point spectrum iff the $\hat{A}$-genus of its compact dual does not vanish. In this case, if $M$ is irreducible, then $M=\mathrm{U}(p, q) / \mathrm{U}(p) \times \mathrm{U}(q)$ with $p+q$ odd, and $\operatorname{Spec}_{p}(D)=\{0\}$.
\end{abstract}

\section{INTRODUCTION}

We investigate the existence of point spectrum of the Dirac operator $D$ acting on spinors over a Riemannian symmetric space $M=G / K$ of noncompact type. Following Seifarth's approach in [S], we look at those discrete series representations of $G$ that appear in $L^{2}(\mathcal{S})$, where $\mathcal{S}$ is the spinor bundle over $M$. We find that the existence of point spectrum of $D$ is equivalent to a regularity condition for the half sum $\rho_{\mathfrak{k}}$ of positive roots of $K$, which in turn is equivalent to the nonvanishing of the $\hat{A}$-genus of the compact dual $M^{\prime}$ of $M$. Using the classification of compact symmetric spaces, we finally determine all noncompact symmetric spaces on which the Dirac operator $D$ has point spectrum. We summarize our results:

0.1. Theorem. Let $M$ be a Riemannian symmetric space of noncompact type, and let $D$ be the Dirac operator acting on spinors over $M$. Then the following statements are equivalent:

(1) the point spectrum of $D$ is nonempty;

(2) the point spectrum of $D$ is precisely $\operatorname{Spec}_{p}(D)=\{0\}$; moreover, as a $G$ module, $\operatorname{ker}(D)$ is irreducible and isomorphic to the discrete series representation with Harish-Chandra parameter $\rho_{\mathfrak{k}}$;

(3) the $\hat{A}$-genus of the compact dual of $M$ is nonzero;

(4) each irreducible factor of $M$ is isometric to $\mathrm{U}(p, q) / \mathrm{U}(p) \times \mathrm{U}(q)$, with $p+q$ odd.

Our present note is motivated by the work of several authors on the spectra of Dirac operators on noncompact Riemannian symmetric spaces. Using the Plancherel theorem, Bunke computed the whole spectrum of the untwisted Dirac operator $D$ on the real hyperbolic spaces in $[\mathrm{Bu}]$ (note the incorrect statement concerning the eigenvalue 0). Seifarth showed the nonexistence of point spectrum on

Received by the editors September 18, 2000.

2000 Mathematics Subject Classification. Primary 58C40; Secondary 53C35, 22E30.

Both authors were supported by a research fellowship of the DFG.

(C)2001 American Mathematical Society 
the real and quaternion hyperbolic spaces in [S] (the treatment of the complex hyperbolic space is incomplete). Another computation of the spectrum of $D$ on $\mathbb{R} H^{n}$ by Camporesi and Higuchi uses polar coordinates and separation of variables $([\mathrm{CH}])$. Using a similar approach, Baier proved in [Ba] that the Dirac operator on $\mathbb{C} H^{n}$ has no eigenvalue $\lambda$ with $|\lambda| \geq \frac{n-1}{4}$. Let us also mention the results of Galina and Vargas on the eigenvalues of twisted Dirac operators: In [GV], they compute the spectrum of Dirac operators on $\mathbb{R} H^{n}$ and $\mathbb{C} H^{n}$, twisted with a homogeneous vector bundle. They consider only the case where the inducing $K$-representation has a sufficiently nonsingular highest weight.

The rest of this paper is organized as follows: In Section 1, we recall the relation between point spectrum of homogeneous selfadjoint elliptic operators on $M=G / K$ and discrete series representations of $G$. In Section 2, we show that the existence of point spectrum of $D$ on $M$ is equivalent to the nonvanishing of the $\hat{A}$-genus on the compact dual of $M$. Finally, in Section 3 , we classify the compact symmetric spaces $M^{\prime}$ with $\hat{A}\left(M^{\prime}\right)\left[M^{\prime}\right] \neq 0$.

This work was written while the second named author enjoyed the hospitality and support of the IHES (Bures-sur-Yvette). The first named author would like to thank the Université de Paris-Sud (Orsay) for its hospitality. We are grateful to C. Bär, J.-M. Bismut and W. Müller for helpful discussions. We wish to thank M. Olbrich for carefully reading the manuscript, pointing out a few inaccuracies, and suggesting an alternative proof of Corollary 2.12 .

\section{The POINT SPECTRUM AND THE DISCRETE SERIES}

Let $M=G / K$ be a Riemannian symmetric space of noncompact type. Here, $G$ is a noncompact connected semisimple Lie group, and $K$ is a maximal compact subgroup. We fix a $G$-invariant metric on $M$. Then $M$ is a Hadamard manifold, i.e., the Riemannian exponential map exp: $T_{p} M \rightarrow M$ is a diffeomorphism at each point $p$ of $M$. In particular, $M$ is contractible, and thus possesses a unique spin structure.

Let $\mathfrak{g}=\mathfrak{k} \oplus \mathfrak{p}$ be the Cartan decomposition of $\mathfrak{g}$, where $\mathfrak{k}$ is the Lie algebra of $K$. A homogeneous spin structure can be described by a lift $\tilde{\alpha}$ of the adjoint representation $\alpha: K \rightarrow \mathrm{SO}(\mathfrak{p})$ to $\operatorname{Spin}(\mathfrak{p})$. We can assume the existence of such a lift (if necessary, we replace $G$ and $K$ by suitable double covers). The (complex) spin representation $(\rho, S)$ of $\operatorname{Spin}(\mathfrak{p})$ gives rise to a $K$-representation $(\sigma, S)$, with $\sigma:=$ $\rho \circ \tilde{\alpha}$. The spinor bundle is then isomorphic to the homogeneous vector bundle

$$
\mathcal{S}:=G \times_{\sigma} S
$$

induced by $\sigma$. The Levi-Civita connection on $M$ induces a connection on $\mathcal{S}$.

Let $\Gamma_{c}(\mathcal{S})$ be the space of compactly supported smooth sections of $\mathcal{S}$, and let $L^{2}(\mathcal{S})$ be its Hilbert space completion. The Dirac operator acts on $\Gamma_{c}(\mathcal{S})$ as the composition of covariant derivative and Clifford multiplication. Since $M=G / K$ is a complete manifold, the Dirac operator is essentially selfadjoint (cf. [W]). Hence, its minimal and maximal closed extension coincide. Let $D$ be the unique selfadjoint extension to a closed operator. It commutes with the natural action of $G$ on $L^{2}(\mathcal{S})$. More generally, one can consider Dirac operators on $L^{2}(\mathcal{S} \otimes \mathcal{W})$, where $\mathcal{W}$ is a homogeneous Hermitian vector bundle over $M$ which is equipped with an equivariant unitary connection. 
Because $D$ is selfadjoint, its spectrum consists only of point spectrum and continuous spectrum. Moreover, it is completely contained in $\mathbb{R}$. The point spectrum $\operatorname{Spec}_{p}(D)$, i.e., the set of eigenvalues, is defined as

$$
\operatorname{Spec}_{p}(D):=\{\lambda \in \mathbb{C} \mid \operatorname{ker}(D-\lambda) \neq\{0\}\} .
$$

If $\lambda$ is an eigenvalue of $D$, the dimension of the eigenspace $\operatorname{ker}(D-\lambda)$ is called the multiplicity of $\lambda$.

Clearly, $G$ acts on the eigenspaces of $D$. It turns out that the eigenspaces are direct sums of irreducible $G$-representations belonging to the discrete series:

1.2. Definition. An irreducible representation $(\pi, H)$ of $G$ is called a discrete series representation iff the matrix coefficients $g \mapsto\langle\pi(g) v, w\rangle$ for all $v, w \in H$ are square integrable on $G$ with respect to the Haar measure. Let $\hat{G}_{d}$ be the set of equivalence classes of discrete series representations.

The main tool of our investigation of $\operatorname{Spec}_{p}(D)$ is the following

1.3. Theorem (cf. [AS], CM]). Let $D$ be a homogeneous selfadjoint elliptic differential operator on $\mathcal{E}:=G \times_{\varepsilon} E$ for some $K$-representation $(\varepsilon, E)$. Then the direct sum of all eigenspaces of $D$ is isomorphic to

$$
\bigoplus_{\pi \in \hat{G}_{d}} \pi \otimes \operatorname{Hom}_{K}\left(\left.\pi\right|_{K}, \varepsilon\right) \text {. }
$$

In particular, a discrete series representation $\pi$ of $G$ is isomorphic to a subrepresentation of $L^{2}(\mathcal{E})$ iff $\left.\pi\right|_{K}$ has an irreducible $K$-subrepresentation in common with $\varepsilon$. In this case, we say that $\pi \in \hat{G}_{d}$ contributes to $\operatorname{Spec}_{p}(D)$.

Proof of Theorem 1.3. Since $D$ is a $G$-invariant elliptic differential operator, we can apply a theorem of Connes and Moscovici ( $\mathrm{CM}$, Theorem 6.1). It follows that each eigenspace of $D$ is isomorphic to a finite sum of discrete series representations of $G$.

On the other hand, by the Plancherel Theorem and Frobenius reciprocity (cf. [AS], Chapter 2), we have

$$
\operatorname{Hom}_{G}\left(\pi, L^{2}(\mathcal{E})\right) \cong \operatorname{Hom}_{K}\left(\left.\pi\right|_{K}, \varepsilon\right)
$$

for each discrete series representation $\pi$. Also, $D$ is $G$-invariant and $\operatorname{Hom}_{K}\left(\left.\pi\right|_{K}, \varepsilon\right)$ is finite dimensional by results of Harish-Chandra (Theorem 8.1 in $[\mathrm{K}]$ ). Hence, it is easy to check that

$$
\pi \otimes \operatorname{Hom}_{G}\left(\pi, L^{2}(\mathcal{E})\right) \subset L^{2}(\mathcal{E})
$$

decomposes as a finite sum of $D$-eigenspaces.

Each eigenvalue has infinite multiplicity, since all nontrivial unitary representations of a noncompact connected semisimple Lie group are infinite dimensional. Moreover, if $D$ has nonempty point spectrum, then $G$ has discrete series representations. Due to a theorem of Harish-Chandra (cf. [K], Theorem 12.20, [AS], Proposition 6.11), this is the case iff $\operatorname{rk}(G)=\operatorname{rk}(K)$. Hence, we have the following

1.4. Remark. On a noncompact symmetric space $G / K$ with $\operatorname{rk}(G)>\operatorname{rk}(K)$, the point spectrum of the Dirac operator $D$ is empty. 


\section{Minimal $K$-Types AND POINT SPECTRUm}

In this section, we recall a few facts from the theory of discrete series representations of $G$. We will show that at most one irreducible subrepresentation of $\sigma$ can occur as a $K$-type of a discrete series representation of $G$. This happens iff the half sum $\rho_{\mathfrak{k}}$ of positive roots of $K$ is $\mathfrak{g}$-regular. Using this, we prove the equivalence of statements (1) - (3) of Theorem 0.1. We remark that our arguments in this section are also valid for nonirreducible symmetric spaces.

a) Discrete series representations and their $K$-types. By Remark 1.4 , we may and will assume from now on that $\operatorname{rk}(G)=\operatorname{rk}(K)$. Then we fix a common maximal torus $H \subset K \subset G$ with Lie algebra $\mathfrak{h}$ and weight lattice

$$
\Gamma:=\left\{\gamma \in i \mathfrak{h}^{*} \mid \gamma(X) \in 2 \pi i \mathbb{Z} \text { for all } X \in \mathfrak{h} \text { with } e^{X}=e\right\} .
$$

Let $\Delta_{\mathfrak{g}}=\Delta_{\mathfrak{k}} \cup \Delta_{\mathfrak{p}}$ be the root system of $G$ with respect to $\mathfrak{h}$, decomposed into the root system of $K$ and the set of noncompact roots. Choose systems of positive roots $\Delta_{\mathfrak{g}}^{+} \supset \Delta_{\mathfrak{k}}^{+}$, and let $P_{\mathfrak{g}} \subset P_{\mathfrak{k}} \subset i \mathfrak{h}^{*}$ be the Weyl chambers associated to $\Delta_{\mathfrak{g}}^{+}$ and $\Delta_{\mathfrak{k}}^{+}$. Let $W_{\mathfrak{g}}, W_{\mathfrak{k}}$ be the Weyl groups of $G$ and $K$, and set

$$
W^{\prime}:=\left\{w \in W_{\mathfrak{g}} \mid w\left(P_{\mathfrak{g}}\right) \subset P_{\mathfrak{k}}\right\} .
$$

Let $\rho_{\mathfrak{g}}$ and $\rho_{\mathfrak{k}}$ be the half sums of positive roots of $G$ and $K$.

We fix an $A d_{K}^{*}$-invariant scalar product on $\mathfrak{k}^{*}$. We call a weight $\lambda \in i \mathfrak{h}^{*} \mathfrak{g}$ regular if $\langle\lambda, \gamma\rangle \neq 0$ for all $\gamma \in \Delta_{\mathfrak{g}}$, and $\mathfrak{g}$-singular otherwise. If $\langle\lambda, \gamma\rangle \neq 0$ holds only for $\gamma \in \Delta_{\mathfrak{k}}$, then $\lambda$ is called $\mathfrak{k}$-regular. Clearly, $\mathfrak{g}$-regularity implies $\mathfrak{k}$-regularity. An element $\kappa \in i \mathfrak{h}^{*}$ is called $\mathfrak{k}$-algebraically integral iff

$$
2 \frac{\langle\alpha, \kappa\rangle}{\langle\alpha, \alpha\rangle} \in \mathbb{Z}
$$

for all $\alpha \in \Delta_{\mathfrak{k}}^{+}$. Note that all weights of $K$, i.e. all elements of $\Gamma$, are automatically $\mathfrak{k}$-algebraically integral. Note also that $\rho_{\mathfrak{k}}$ and $\rho_{\mathfrak{g}}$ are $\mathfrak{k}$-algebraically integral (for $\rho_{\mathfrak{k}}$ this is well known, for $\rho_{\mathfrak{g}}$ it follows because $\rho_{\mathfrak{g}}$ is $\mathfrak{g}$-algebraically integral). Furthermore, $\rho_{\mathfrak{k}}$ uniquely minimizes $|\kappa|$ among all $\mathfrak{k}$-algebraically integral $\mathfrak{k}$-regular elements $\kappa$ of $P_{\mathfrak{k}}$ (for semisimple $K$, this is well known, in the general case it follows because the center of $K$ is orthogonal to its semisimple part).

Let us now turn to some facts about the discrete series of $G$, in particular about the possible $K$-types.

2.1. Definition. Let $\pi \in \hat{G}_{d}$ be a discrete series representation of $G$, and let $\varphi_{\kappa}$ be an irreducible representation of $K$ with highest weight $\kappa \in \Gamma \cap P_{\mathfrak{k}}$. Then $\kappa$ is called a $K$-type of $\pi$, if $\left.\pi\right|_{K}$ contains an irreducible subrepresentation isomorphic to $\varphi_{\kappa}$. The dimension of $\operatorname{Hom}_{K}\left(\pi, \varphi_{\kappa}\right)$ is called the multiplicity of $\kappa$.

If $\kappa$ minimizes $\left|\kappa+2 \rho_{\mathfrak{k}}\right|$ among all $K$-types, then $\kappa$ is called a minimal $K$-type of $\pi$.

Our argumentation is based upon the following fundamental result of HarishChandra:

2.2. Theorem ([AS], Theorems 8.1 and $8.5,[\mathrm{~K}]$, Theorems 9.20 and 12.21). The discrete series representations of $G$ are parametrized by $\lambda \in P_{\mathfrak{k}}$ with $\lambda \in w\left(P_{\mathfrak{g}}\right)$ for some $w \in W^{\prime}$ such that $\lambda$ is regular and $\lambda-w \rho_{\mathfrak{g}} \in \Gamma$. For such a $\lambda$, the discrete series representation $\pi_{\lambda}$ corresponding to $\lambda$ has a unique minimal $K$-type

$$
\kappa:=\lambda+w \rho_{\mathfrak{g}}-2 \rho_{\mathfrak{k}},
$$


which occurs with multiplicity 1. Finally, each $K$-type $\kappa^{\prime}$ of $\pi_{\lambda}$ is of the form

$$
\kappa^{\prime}:=\kappa+\sum_{\substack{\alpha \in \Delta_{\mathfrak{g}} \\\left\langle w \rho_{\mathfrak{g}}, \alpha\right\rangle>0}} n_{\alpha} \alpha
$$

where the $n_{\alpha}$ are nonnegative integers.

In the literature, $\lambda$ is called the Harish-Chandra parameter for $\pi_{\lambda}$, while $\kappa$ is called the Blattner parameter.

b) The Dirac operator on spinors. Let $\mathcal{S}=G \times{ }_{\sigma} S$ be the spinor bundle on $M$ as in (1.1). Since we assume that $\operatorname{rk}(G)=\operatorname{rk}(K)$, the symmetric space $M$ is even dimensional. In particular, the spinor representation and the spinor bundle split into a positive and a negative part:

$$
S=S^{+} \oplus S^{-} \quad \text { and } \quad \mathcal{S}=\mathcal{S}^{+} \oplus \mathcal{S}^{-} .
$$

The $K$-action on $S^{+}$and $S^{-}$is described by a formula of Parthasarathy:

2.4. Lemma $\left([\mathrm{P}]\right.$, Lemma 2.2)). For $w \in W^{\prime}$, let $\sigma^{w \rho_{\mathfrak{g}}-\rho_{\mathfrak{k}}}$ be the $K$-representation with highest weight $w \rho_{\mathfrak{g}}-\rho_{\mathfrak{k}} \in P_{\mathfrak{k}}$. Then for a suitable orientation of $M, \sigma$ decomposes as

$$
\sigma=\sigma^{+} \oplus \sigma^{-}:=\bigoplus_{\substack{w \in W^{\prime} \\
\operatorname{sign}(w)=1}} \sigma^{w \rho_{\mathfrak{g}}-\rho_{\mathfrak{k}}} \oplus \bigoplus_{\begin{array}{c}
w \in W^{\prime} \\
\operatorname{sign}(w)=-1
\end{array}} \sigma^{w \rho_{\mathfrak{g}}-\rho_{\mathfrak{k}}}
$$

2.5. Remark. This implies in particular that $\rho_{\mathfrak{k}}-w \rho_{\mathfrak{g}} \in \Gamma$, because we have assumed that $\sigma$ is a representation of $K$. Note that $w \in W_{\mathfrak{g}}$ may be arbitrary, because different $W_{\mathfrak{g}}$-translates differ by linear combinations of roots of $G$, which are clearly in $\Gamma$.

2.6. Remark. We recall that the operator $D$ splits as

$$
D^{ \pm}:=\left.D\right|_{\Gamma\left(\mathcal{S}^{ \pm}\right)}: \Gamma\left(\mathcal{S}^{ \pm}\right) \rightarrow \Gamma\left(\mathcal{S}^{\mp}\right) \text {. }
$$

If $E_{\mu}$ is an eigenspace corresponding to an eigenvalue $\mu$ of $D^{2}$, then $E_{\mu}$ splits into $E_{\mu}^{+} \oplus E_{\mu}^{-}$, with $E_{\mu}^{ \pm}:=E_{\mu} \cap \Gamma\left(\mathcal{S}^{ \pm}\right)$. If, moreover, $\mu \neq 0$, then $\left.D^{ \pm}\right|_{E_{\mu}^{ \pm}}: E_{\mu}^{ \pm} \rightarrow E_{\mu}^{\mp}$ is an isomorphism.

We will now establish an algebraic criterion for the existence of point spectrum for untwisted Dirac operators.

2.7. Theorem. Let $D$ be the untwisted Dirac operator on $M=G / K$. If $\rho_{\mathfrak{k}}$ is $\mathfrak{g}$-regular, then $\operatorname{Spec}_{p}(D)=\{0\}$, and $\operatorname{ker}(D)$ is isomorphic to the discrete series representation with Harish-Chandra parameter $\rho_{\mathfrak{k}}$. If $\rho_{\mathfrak{k}}$ is $\mathfrak{g}$-singular, then there is no point spectrum.

Remark. By $\left[\mathrm{AS}\right.$, Theorem 9.3, we already know that $\operatorname{ker}(D) \neq 0$ iff $\rho_{\mathfrak{k}}$ is $\mathfrak{g}$-regular. It would thus be enough to check that $\operatorname{Spec}_{p}(D)$ contains no nonzero eigenvalues.

Proof. First of all, if $\rho_{\mathfrak{k}}$ is $\mathfrak{g}$-regular, then there exists a discrete series representation with Harish-Chandra parameter $\rho_{\mathfrak{k}}$ because of Theorem 2.2 and Remark 2.5. Let $w \in W^{\prime}$ such that $\rho_{\mathfrak{k}} \in w\left(P_{\mathfrak{g}}\right)$. The minimal $K$-type of $\pi_{\rho_{\mathfrak{k}}}$ is $w \rho_{\mathfrak{g}}-\rho_{\mathfrak{k}}$, which is a highest weight of $\sigma$ by Lemma 2.4. Hence by Theorem 1.3. $D$ has point spectrum.

On the other hand, let us assume that $\pi_{\lambda}$ is a discrete series representation of $G$ that contributes to $\operatorname{Spec}_{p}(D)$. We will show that then necessarily $\lambda=\rho_{\mathfrak{k}}$. 
Let $w \in W^{\prime}$ be such that $\lambda \in w\left(P_{\mathfrak{g}}\right)$. By Theorem 1.3 and Lemma [2.4, for some $w_{0} \in W^{\prime}$, the weight $w_{0} \rho_{\mathfrak{g}}-\rho_{\mathfrak{k}}$ is a $K$-type of $\pi_{\lambda}$. Then by Theorem $[2.2$ there exist nonnegative integers $n_{\alpha}$ such that

$$
w_{0} \rho_{\mathfrak{g}}+\rho_{\mathfrak{k}}=\lambda+w \rho_{\mathfrak{g}}+\sum_{\substack{\alpha \in \Delta_{\mathfrak{g}}^{+} \\\left\langle w \rho_{\mathfrak{g}}, \alpha\right\rangle \geq 0}} n_{\alpha} \alpha .
$$

We establish a few inequalities: By construction, $\lambda$ and $w \rho_{\mathfrak{g}}$ are both $\mathfrak{g}$-regular and lie in the same Weyl chamber $w\left(P_{\mathfrak{g}}\right)$ of $\mathfrak{g}$. This has two consequences: First, the weight $w \rho_{\mathfrak{g}}$ uniquely minimizes the distance to $\lambda$ among all $W_{\mathfrak{g}}$-translates of $\rho_{\mathfrak{g}}$. Thus

$$
\left\langle\lambda, w_{0} \rho_{\mathfrak{g}}\right\rangle \leq\left\langle\lambda, w \rho_{\mathfrak{g}}\right\rangle,
$$

with equality iff $w_{0}=w$. Second, $\left\langle\alpha, w \rho_{\mathfrak{g}}\right\rangle>0$ iff $\langle\alpha, \lambda\rangle>0$. This implies

$$
\left\langle\lambda, \sum n_{\alpha} \alpha\right\rangle \geq 0
$$

since the $n_{\alpha}$ have to be nonnegative. Moreover, we have equality iff all the $n_{\alpha}$ are zero.

If (2.8) holds for $\lambda$, then $\lambda$ must clearly be $\mathfrak{k}$-algebraically integral, because the same holds for $\rho_{\mathfrak{k}}, \rho_{\mathfrak{g}}$ and all $\alpha \in \Delta_{\mathfrak{g}}^{+}$. Now the weight $\rho_{\mathfrak{k}}$ uniquely minimizes $|\kappa|$ among all $\mathfrak{k}$-regular $\mathfrak{k}$-algebraically integral $\kappa \in P_{\mathfrak{k}}$. Because $\lambda$ is $\mathfrak{g}$-regular, it is also $\mathfrak{k}$-regular, and we have

$$
\left\langle\rho_{\mathfrak{k}}, \lambda\right\rangle \leq|\lambda| \cdot\left|\rho_{\mathfrak{k}}\right| \leq|\lambda|^{2}
$$

with equality iff $\lambda=\rho_{\mathfrak{k}}$.

In order to show that $\lambda=\rho_{\mathfrak{k}}$, we multiply (2.8) by $\lambda$ and apply (2.9) and (2.10):

$$
\left\langle w_{0} \rho_{\mathfrak{g}}+\rho_{\mathfrak{k}}, \lambda\right\rangle=\left\langle\lambda+w \rho_{\mathfrak{g}}+\sum n_{\alpha} \alpha, \lambda\right\rangle \quad \Longrightarrow \quad\left\langle\rho_{\mathfrak{k}}, \lambda\right\rangle \geq|\lambda|^{2} .
$$

So by (2.11), we have equality, which means that $\lambda=\rho_{\mathfrak{k}}$, that $w_{0}=w$, and that all $n_{\alpha}$ are zero. Now, $\rho_{\mathfrak{k}}$ can only be a Harish-Chandra parameter for a discrete series representation of $G$ if $\rho_{\mathfrak{k}}$ is $\mathfrak{g}$-regular. Thus, there is no point spectrum if $\rho_{\mathfrak{k}}$ is $\mathfrak{g}$-singular.

Let us assume that $\rho_{\mathfrak{k}}$ is $\mathfrak{g}$-regular. Then, among the highest weights of $\sigma$, only $w \rho_{\mathfrak{g}}-\rho_{\mathfrak{k}}$ can appear as a $K$-type of a discrete series representation of $G$. This implies that the eigenspaces of $D^{2}$ are contained either in $L^{2}\left(\mathcal{S}^{+}\right)$or in $L^{2}\left(\mathcal{S}^{-}\right)$. In particular, $\operatorname{Spec}_{p}\left(D^{2}\right) \subset\{0\}$, because by Remark 2.6, any nonzero eigenvalue $\mu$ would lead to an eigenspace $E_{\mu}=E_{\mu}^{+} \oplus E_{\mu}^{-}$with $E_{\mu}^{+} \cong E_{\mu}^{-} \approx\{0\}$. Finally, $w \rho_{\mathfrak{g}}-\rho_{\mathfrak{k}}$ is the minimal $K$-type of $\pi_{\rho_{\mathfrak{k}}}$. Hence, it has multiplicity 1 , and $\operatorname{ker}(D)=\operatorname{ker}\left(D^{2}\right)$ is irreducible as a $G$-module. The isomorphism $\operatorname{ker}(D)=\operatorname{ker}\left(D^{2}\right)$ is of course well known, e.g., as Theorem 5.7 in [LM].

Remark. Another way to check that $D$ vanishes on $\pi_{\rho_{\mathfrak{k}}} \subset L^{2}(\mathcal{S})$ is to express $D^{2}$ in terms of the Casimir operator $\Omega$ of $G([\mathrm{P}$, Proposition 3.1, [K], Lemma 12.12), and using the explicit formula for $\pi_{\lambda}(\Omega)$ ([K], Lemma 12.28).

We will now reformulate the theorem above in terms of the compact dual of $M$. Therefore, let $\mathfrak{g}^{\mathbb{C}}$ be the complexification of $\mathfrak{g}$. Recall that there exists a compact, connected, simply connected Lie group $G^{\prime}$ with Lie algebra $\mathfrak{g}^{\prime}:=\mathfrak{k} \oplus i \mathfrak{p}$. Let $K^{\prime} \subset G^{\prime}$ be its Lie subgroup with Lie algebra $\mathfrak{k}$; then $K^{\prime}$ is closed, and $M^{\prime}:=G^{\prime} / K^{\prime}$ is called 
the compact dual of $M$. Note that $\mathfrak{h}$ is a common Cartan subalgebra of $\mathfrak{g}, \mathfrak{g}^{\prime}$ and $\mathfrak{k}$, and that $\mathfrak{g}$ and $\mathfrak{g}^{\prime}$ have the same roots, Weyl chambers, etc. with respect to $\mathfrak{h}$.

With these definitions, we can give an equivalent criterion for the existence of point spectrum:

2.12. Corollary. Let $D$ be the untwisted Dirac operator on $M=G / K$. Then $D$ has point spectrum iff the $\hat{A}$-genus of the compact dual $M^{\prime}=G^{\prime} / K^{\prime}$ of $M$ is nonzero.

Proof. By $[\mathrm{BH}]$, the $\hat{A}$-genus of $M^{\prime}$ is given by the formula

$$
\hat{A}\left(M^{\prime}\right)\left[M^{\prime}\right]=\prod_{\alpha \in \Delta_{\mathfrak{g}}} \frac{\left\langle\alpha, \rho_{\mathfrak{k}}\right\rangle}{\left\langle\alpha, \rho_{\mathfrak{g}}\right\rangle}
$$

for a suitable orientation of $M^{\prime}$. In particular, $\hat{A}\left(M^{\prime}\right)\left[M^{\prime}\right] \neq 0$ iff $\rho_{\mathfrak{k}}$ is $\mathfrak{g}$-regular; cf. Theorem 23.3 in $[\mathrm{BH}]$. Thus, our claim follows from Theorem 2.7

Remark. The following alternative proof motivates the appearance of the $\hat{A}$-genus: By Theorem 2.7, $D$ has point spectrum iff its $L^{2}$-index is nonzero. Using Hirzebruch proportionality, one then concludes that this is the case iff $\hat{A}\left(M^{\prime}\right)\left[M^{\prime}\right] \neq 0$ (cf. [AS], Chapter 3 and erratum). This was suggested by M. Olbrich.

Let us state some consequences of our criterion.

2.14. Corollary. Let $D$ be the untwisted Dirac operator on $M=G / K$. If $D$ has point spectrum, then

(1) $M$ is a Hermitian symmetric space,

(2) the compact dual $M^{\prime}$ of $M$ carries no spin structure, and

(3) the dimension of $M$ is divisible by 4.

Proof. By [BH], Theorem 23.3, $M^{\prime}$ is Hermitian symmetric if its $\hat{A}$-genus is nonzero. Then $M$ is also Hermitian symmetric. Next, $M^{\prime}$ has positive scalar curvature. Thus if $M^{\prime}$ was spin, its $\hat{A}$-genus would vanish by Lichnerowicz' theorem ([LM], Corollary 8.9). Finally, the $\hat{A}$-genus of $M^{\prime}$ can be nonzero only if $\operatorname{dim} M^{\prime}$ is divisible by 4 . Hence, the claims follows from Corollary 2.12

Remark. The conditions listed in Corollary 2.14 are not sufficient for the existence of point spectrum: In the next section, we will see that, for $M^{\prime}:=\mathrm{SP}(n) / \mathrm{U}(n)$ with $n \in 4 \mathbb{N}$, conditions (1) - (3) above are satisfied. Nevertheless, the $\hat{A}$-genus of $M^{\prime}$ vanishes.

\section{Compact symmetric SPaCes With NONVANishing $\hat{A}$-genus}

In this section we want to determine the compact Riemannian symmetric spaces $M^{\prime}=G^{\prime} / K^{\prime}$ with nonvanishing $\hat{A}$-genus. By [Bo], we may again assume that $\operatorname{rk}\left(G^{\prime}\right)=\operatorname{rk}\left(K^{\prime}\right)$. Because the $\hat{A}$-genus is multiplicative on products of manifolds, we restrict our attention to irreducible symmetric spaces. By Corollary 2.14, we only have to investigate compact Hermitian symmetric spaces $M^{\prime}$ with $\operatorname{dim} M^{\prime} \in 4 \mathbb{N}$ which are not spin.

The simply connected symmetric spaces that admit a spin structure are known (cf. [CG] or [HS]). On the other hand there are four families of Hermitian symmetric spaces and two exceptional ones (cf. $[\mathrm{H}]$ ). Combining these lists, we see that the 
irreducible Hermitian symmetric spaces which have no spin structure form the following three families:

$$
\begin{array}{cl}
\mathrm{SO}(n+2) / \mathrm{SO}(2) \times \mathrm{SO}(n) & \text { for } n \text { odd, } \\
\mathrm{SP}(n) / \mathrm{U}(n) & \text { for } n \text { even, and } \\
\mathrm{U}(p+q) / \mathrm{U}(p) \times \mathrm{U}(q) & \text { for } p+q \text { odd }
\end{array}
$$

(in the last case, $M$ should actually be represented as a quotient of a finite cover of $\mathrm{SU}(p, q)$, rather than of $\mathrm{U}(p, q))$. We will show that all manifolds of the families (1) and (2) have vanishing $\hat{A}$-genus, while the $\hat{A}$-genus of $\mathrm{U}(p+q) / \mathrm{U}(p) \times \mathrm{U}(q)$ is different from zero if $p+q$ is odd.

The manifolds of family (1) have dimension $2 n$. Since we assume that $n$ is odd, the dimension is not divisible by 4 , and the $\hat{A}$-genus vanishes. For the two other families, we have to use formula (2.13) and to compute the scalar products $\left\langle\alpha, \rho_{\mathfrak{k}}\right\rangle$ for all positive roots $\alpha \in \Delta_{\mathfrak{g}}^{+}$.

Let us investigate family (2). The Lie algebra of $\operatorname{SP}(n)$ is the Lie algebra of skewHermitian quaternionic matrices of order $n$. The Lie algebra of $\mathrm{U}(n)$ is realized as the sub-algebra of skew-Hermitian complex matrices of order $n$. A common Cartan sub-algebra $\mathfrak{h}$ is the Lie algebra of the matrices of the form

$$
\lambda=\left(\lambda_{1}, \ldots, \lambda_{n}\right):=\operatorname{diag}\left(\lambda_{1}, \ldots, \lambda_{n}\right),
$$

with $\lambda_{j} \in i \mathbb{R}$. As a system of positive roots of $\operatorname{SP}(n)$, we take

$$
\Delta_{\mathfrak{g}}^{+}=\left\{\lambda_{i} \pm \lambda_{j} \mid 1 \leq i<j \leq n\right\} \cup\left\{2 \lambda_{i} \mid i=1, \ldots, n\right\} .
$$

The positive roots in $K^{\prime}=\mathrm{U}(n)$ are $\Delta_{\mathfrak{k}}^{+}:=\left\{\lambda_{i}-\lambda_{j} \mid i<j\right\}$. Therefore, $\rho_{\mathfrak{k}}=$ $(n-1, n-3, \ldots, 1-n)$. Clearly, the standard scalar product on $\mathfrak{h}^{*} \cong \mathbb{R}^{n}$ extends to an $A d_{G^{\prime}}^{*}$-invariant scalar product on $\mathfrak{g}^{\prime *}$. In particular, for $\alpha=\left(\lambda_{1}+\lambda_{n}\right)$ we have $\left\langle\alpha, \rho_{\mathfrak{k}}\right\rangle=0$. Hence, according to formula (2.13), the $\hat{A}$-genus of all manifolds $\mathrm{SP}(n) / \mathrm{U}(n)$ is zero.

The computation for family (3) is similar. As a system of positive roots we take

$$
\Delta_{\mathfrak{g}}^{+}=\left\{\lambda_{i}-\lambda_{j} \mid 1 \leq i<j \leq p+q\right\} .
$$

The positive roots in $K^{\prime}=\mathrm{U}(p) \times \mathrm{U}(q)$ are

$$
\Delta_{\mathfrak{k}}^{+}=\left\{\lambda_{i}-\lambda_{j} \mid 1 \leq i<j \leq p\right\} \cup\left\{\lambda_{i}-\lambda_{j} \mid p+1 \leq i<j \leq p+q\right\} .
$$

This yields $\rho_{\mathfrak{k}}=(p-1, p-3, \ldots, 1-p, q-1, q-3, \ldots, 1-q)$. Again we take as a scalar product for the roots the canonical scalar product of vectors in $\mathbb{R}^{n}$. Since $p+q$ is odd, we can assume $p$ to be even and $q$ to be odd. Hence, all numbers $p-1, p-3$, $\ldots, 1-p$ are odd and all numbers $q-1, q-3, \ldots, 1-q$ are even. From this it follows that the scalar product $\left\langle\alpha, \rho_{\mathfrak{k}}\right\rangle$ for any positive root $\alpha \in \Delta_{\mathfrak{g}}^{+}$is different from zero. Using once again formula (2.13), we obtain that the $\hat{A}$-genus of $\mathrm{U}(p+q) / \mathrm{U}(p) \times \mathrm{U}(q)$ is nonzero.

In particular, the $\hat{A}$-genus of the complex projective space $\mathbb{C} P^{2 n}$ does not vanish. Here a simple computation gives $\hat{A}\left(\mathbb{C} P^{2 n}\right)\left[\mathbb{C} P^{2 n}\right]=(-4)^{-n} \prod_{i=1}^{n} \frac{2 i-1}{2 i}=$ $(-16)^{-n}\left(\begin{array}{c}2 n \\ n\end{array}\right)$. Finally, we have

3.1. Theorem. Let $M^{\prime}=G^{\prime} / K^{\prime}$ be an irreducible Riemannian symmetric space of compact type. Then $M^{\prime}$ has nonvanishing $\hat{A}$-genus iff $M^{\prime}$ is isometric to

$$
\mathrm{U}(p+q) / \mathrm{U}(p) \times \mathrm{U}(q), \quad \text { with } p+q \text { odd } .
$$


Together with Theorem [2.7, Corollary [2.12, and the multiplicativity of $\hat{A}$, this proves Theorem 0.1 .

\section{REFERENCES}

[AS] M. F. Atiyah, W. Schmid, A Geometric Construction of the Discrete Series for Semisimple Lie Groups, Inv. math. 42 (1977), 1-62; Erratum, Inv. math. 54 (1979), 189-192. MR 57:3310 MR 81d:22015

[Ba] P. D. Baier, Über den Diracoperator auf Mannigfaltigkeiten mit Zylinderenden, Diplomarbeit, Universität Freiburg (1997).

[Baer] C. Bär, The Dirac fundamental tone of the hyperbolic space, Geom. Dedicata 41 (1992), 103-107. MR 93c:58217

[BGV] M. Berline, E. Getzler, N. Verne, Heat kernels and Dirac operators, Springer, BerlinHeidelberg-New York (1992).

[BH] A. Borel, F. Hirzebruch, Characteristic Classes and Homogeneous Spaces, II, Amer. J. Math. 81 (1959), 315-382. MR 22:988

[Bo] R. Bott, The Index Theorem for Homogeneous Differential Operators, in S. S. Cairns: Differential and Combinatorial Topology, a Symposium in Honor of Marston Morse, Princeton Univ. Press (1965), 167-186. MR 31:6246

[Bu] U. Bunke, The spectrum of the Dirac operator on the hyperbolic space, Math. Nachr. 153 (1991), 179-190. MR 92h:58196

[CG] M. Cahen, S. Gutt, Spin Structures on Compact Simply Connected Riemannian Symmetric Spaces, Simon Stevin 62 (1988), 209-242. MR 90d:58161

[C] R. Camporesi, The spinor heat kernel in maximally symmetric spaces, Comm. Math. Phys. 148 (1992), 283-308. MR 93g:58140

$[\mathrm{CH}]$ R. Camporesi, A. Higuchi, On the eigenfunctions of the Dirac operator on spheres and real hyperbolic spaces, J. Geom. Phys. 20 (1996), 1-18. MR 98c:58169

[CM] A. Connes, H. Moscovici, The $L^{2}$-Index Theorem for Noncompact Homogeneous Spaces of Lie Groups, Ann. of Math. 115 (1982), 291-330. MR 84f:58108

[FJO] M. Flensted-Jensen, K. Okamoto, An explicit construction of the $K$-finite vectors in the discrete series for an isotropic semisimple symmetric space, Mem. Soc. Math. France, Nouv. sér. 15 (1984), 157-199. MR 87c:22025

[GV] E. Galina, J. Vargas, Eigenvalues and eigenspaces for the twisted Dirac operator over $\mathrm{SU}(N, 1)$ and Spin $(2 N, 1)$, Trans. Amer. Math. Soc. 345 (1994), 97-113. MR 95a:22009

[H] S. Helgason Differential Geometry, Lie Groups and Symmetric Spaces, Academic Press, New York (1978). MR 80k:53081

[HS] F. Hirzebruch, P. Slodowy, Elliptic genera, involutions and homogeneous spin manifolds, Geom. Ded. 35 (1990), 309-343. MR 92a:57028

[K] A. W. Knapp, Representation Theory of Semisimple Groups, Princeton Univ. Press, Princeton, N. J. (1986). MR 87j:22022

[LM] H. B. Lawson, M.-L. Michelsohn, Spin Geometry, Princeton Univ. Press, Princeton, N. J. (1989). MR 91g:53001

[P] K. R. Parthasarathy, Dirac operator and the discrete series, Ann. of Math. 96 (1972), $1-30$.

[S] S. Seifarth, The discrete spectrum of the Dirac operators on certain symmetric spaces, Preprint No. 25 of the IAAS, Berlin (1992).

[W] J. A. Wolf, Essential self-adjointness for the Dirac operator and its square, Indiana Univ. Math. J. 22 (1972/73), 611-640. MR 46:10340

Mathematisches Institut, Universität Tübingen, Auf der Morgenstelle 10, 72076 Tübingen, Germany

E-mail address: goette@blaschke.mathematik.uni-tuebingen.de

Mathematisches Institut, Universität München, Theresienstr. 39, D-80333 München, Germany

E-mail address: semmelma@rz.mathematik.uni-muenchen.de 